\title{
Mit „Reflectories“ zukunftsfähige Kompetenzen erwerben
}

Am Beispiel der Corona-Pandemie wird mehr denn je deutlich, dass globale Herausforderungen keinesfalls linear verlaufen, sondern Auswirkungen von Handeln oder Nicht-Handeln äußerst komplex sind und Entscheidungen von einzelnen Akteur/-innen auch in private Bereiche hineinwirken und jede Einzelne bzw. jeden Einzelnen betreffen können. Es wird auch deutlich, dass Entscheidungen häufig mit Unsicherheit behaftet sind. Sie müssen getroffen werden, ohne alle Entscheidungsgrundlagen bzw. die Konsequenzen zu kennen. Ökonomische, ökologische und soziale Auswirkungen sollten bei Entscheidungen genauso berücksichtigt werden, wie unterschiedliche Perspektiven und Wertvorstellungen. Für Lehrende und Lernende bedeutet dies, dass Kompetenzen Globalen Lernens (System-, Bewertungs- und Handlungskompetenz) auch in Zukunft von zentraler Bedeutung sein werden.

Mit Hilfe der sogenannten Reflectories sollen genau diese Kompetenzen gefördert werden (Schrüfer, Brendel et al., 2019). Bei Reflectories (das Wort „Reflectory“ setzt sich zusammen aus den Begriffen „reflect" und „(S)tory“) handelt es sich um Online-Lernarrangements, die unter www.reflectories.de abrufbar sind. Die Lernenden werden in eine Geschichte eingebunden und geraten in eine (Dilemma-)Situation, die sie zum Entscheiden auffordert. Auf Grundlage von Audio-Beiträgen, Videos, Bild- und Textmaterialien, Karten oder Statistiken müssen Handlungsoptionen reflektiert, abgewogen und schließlich Entscheidungen getroffen werden. Wie in der Realität sind diese Entscheidungen meist komplex, beinhalten kontroverse Informationen, unterschiedliche Perspektiven und Interessen und zeichnen sich durch Nicht-Wissen und Unsicherheit aus. Ist eine Entscheidung getroffen, erfährt man sofort die Konsequenzen der Entscheidungen, was wiederum Ausgangspunkt für weitere Herausforderungen und neuen Entscheidungen sein kann. Je nach Entscheidung nimmt die Handlung einen unterschiedlichen Verlauf. Einmal getroffene Entscheidungen können nicht rückgängig gemacht werden. Reflectories sind im Sinne lebenslangen Lernens für alle Altersgruppen einsetzbar. Je nach Intention können die Reflectories in Einzelarbeit, in Partnerarbeit oder in Gruppen durchgeführt werden. Trifft man die Entscheidungen alleine, kann man in seinem eigenen (Lern-)Tempo und nach eigenen Interessen vorgehen. Je größer die Gruppen sind, in denen die Entscheidungen getroffen werden, desto mehr wird man diskutieren, argumentieren und auf verschiedene Perspektiven eingehen müssen. Je nach gewünschter Fokussierung und Zielsetzung stehen auf der Website unterschiedliche Arbeitsaufgaben zur Verfügung. Mit einer eingehenderen Analyse unterschiedlicher Meinungen lassen sich zum Beispiel die zugrundeliegenden Werte, Normen und Interessen herausarbeiten, was zur
Förderung der Bewertungskompetenz beitragen kann. Soll verstärkt Wert auf die Förderung von Systemkompetenz gelegt werden, können zunächst die eigenen Entscheidungswege nachgezeichnet werden. Mithilfe von Concept Maps können diese dann beispielsweise zusammengefügt und Zusammenhänge, Auswirkungen und Wechselwirkungen graphisch dargestellt werden. Auf der Website steht didaktisches Material zur Vertiefung der Reflectories zur Verfügung („Für Lehrkräfte"). Die Materialien sind als Anregung zur Vor- und Nachbereitung gedacht und sollten an die jeweilige Lerngruppe angepasst werden. Der sogenannte „Strukturbaum" bietet einen Überblick über die möglichen Entscheidungswege und Informationsmöglichkeiten der einzelnen Reflectories.

Auf der Homepage sind unterschiedliche Reflectories zu finden. Ein Teil der Reflectories („Projekt Reflectories“) wurde mit Mitteln von Engagement Global entwickelt und von spezialisierten Fachwissenschaftler/-innen auf ihre fachliche Konsistenz überprüft. Ein weiterer Teil umfasst die sogenannten „Community-Reflectories“. Diese wurden von Studierenden, Schüler/-innen und Lehrkräften konzipiert und anschließend in Eigenarbeit technisch umgesetzt. Hierfür steht das sogeannte „Autorentool“ zur Verfügung. Dies ermöglicht es, selbst erstellte Audio, Pdf- und Bilddateien einzubinden und zu einem Reflectory zu verknüpfen. Reflectories, die in Eigenarbeit entwickelt werden, können zunächst in einem selbst gewählten, geschützten Personenkreis durchgeführt werden. Eine Veröffentlichung auf der Website erfolgt nach Anfrage und Prüfung. Eine Anleitung zur Funktionsweise des Autorentools steht zum Download bereit.

Zur Nutzung der auf der Website bereitgestellten Reflectories kann jedes browserfähige (mobile) Endgerät verwendet werden. Darüber hinaus ist ein Internetzugang unabdingbar. Wird in größeren Gruppen gleichzeitig mit unterschiedlichen Reflectories gearbeitet, können Kopfhörer sinnvoll sein. Sollen Reflectories selbst erstellt werden, ist ein Laptop oder ein Rechner zur Programmierung mit dem Autorentool erforderlich, zum Arbeiten mit fertigen Reflectories hingegen genügen Tablets oder Smartphones.

Gabriele Schrüfer, Katja Wrenger, Nina Brendel doi.org/10.31244/zep.2021.03.08

\section{Literatur}

Schrüfer, G., Brendel, N., Zitzelsberger, U. \& Wrenger, K. (2019): „Reflectories “ Nachhaltige Entwicklungsziele für Schülerinnen und Schüler im Geographieunterricht erfahrbar machen. In G. Obermaier (Hrsg.) (2019): Vielfältige Geographien - fachliche und kulturelle Diversität im Unterricht nutzbar machen. (Bd. 10, S. 229-238) Bayreuther Kontaktstudium Geographie. 